\title{
The plasma microRNA levels and their relationship with the general health and functional status in female patients with fibromyalgia syndrome
}

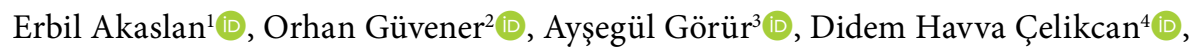 \\ Lülüfer Tamer', Ali Biçer² \\ ${ }^{1}$ Department of Physical Medicine and Rehabilitation, Hatay State Hospital, Hatay, Turkey \\ ${ }^{2}$ Department of Physical Medicine and Rehabilitation, Mersin University Faculty of Medicine, Mersin, Turkey \\ ${ }^{3}$ Department of Medical Biochemistry, Mersin University Faculty of Medicine, Mersin, Turkey \\ ${ }^{4}$ Freelance, Freelance, Mersin, Turkey
}

\begin{abstract}
Objectives: The aim of this study was to identify the plasma level of micro-ribonucleic acid (microRNA) expressions and the relationship between plasma microRNA levels with the general health and functional status in female patients with fibromyalgia syndrome (FMS).

Patients and methods: Thirty-five female patients (mean age: $42.0 \pm 11.8$ years; range, 21 to 62 years) diagnosed as FMS and 35 sex-and age-matched healthy controls (mean age: $43.7 \pm 8.8$ years; range, 21 to 56 years) were enrolled in the study. MicroRNA measurements of the participants in plasma were carried out by using the quantitative polymerase chain reaction (qPCR). A total of 11 plasma levels of microRNA expressions were examined in both groups. The general health and functional status of the patients and controls were assessed by the Fibromyalgia Impact Questionnaire (FIQ) and the Short Form-36 (SF-36) scale.

Results: No significant difference was observed between the plasma levels of microRNA expressions in patients with FMS and healthy controls. The plasma level of miR-320a expression was found to be negatively correlated with the total FIQ score in female patients with $F M S$ ( $p=0.05$, $r=-0.34$ ). Negative correlations were also detected between the plasma level of miR-320a and miR-320b expressions and the subscale score of SF-36 physical function in female patients with FMS $(p=0.01, r=-0.43$ and $p=0.01, r=-0.43$, respectively). A strong positive correlation was found between miR-142-3p and the subscale score of SF-36 mental symptom score in female patients with FMS ( $p<0.001, r=1.00$ ).

Conclusion: The expression levels of microRNAs in plasma between female patients with FMS and controls were not significantly different. Only plasma levels of miR-320a, miR-320b, and miR-142-3p expressions were associated with the general health, functional status, and mental symptom score in female patients with FMS.

Keywords: Fibromyalgia, Fibromyalgia Impact Questionnaire, microRNA, Short Form-36.
\end{abstract}

Fibromyalgia syndrome (FMS) is one of the most common musculoskeletal disorders, affecting predominantly female individuals over than 50 years old worldwide $(0.2$ to $6.6 \%$ of the world population), characterized by several clinical symptoms, including chronic diffuse body pain, increased sensitivity in specific anatomical regions, fatigue or physical exhaustion, sleep disturbances, irritable bowel syndrome (IBS), and cognitive disturbances. Chronic widespread

Received: July 29, 2020 Accepted: February 26, 2021 Published online: October 18, 2021

Correspondence: Orhan Güvener, MD. Mersin Üniversitesi Tıp Fakültesi Fiziksel Tıp ve Rehabilitasyon Anabilim Dalı, 33343 Yenişehir, Mersin, Türkiye. Tel: +90 530 - 2398815 e-mail: dr.orhanguvener@gmail.com

\section{Citation:}

Akaslan E, Güvener O, Görür A, Çelikcan DH, Tamer L, Biçer A. The plasma microRNA levels and their relationship with the general health and functional status in female patients with fibromyalgia syndrome. Arch Rheumatol 2021;36(4):482-492. 
musculoskeletal pain is the most frequent clinical complaint in the vast majority of the patients with FMS. Although chronic pain has been defined as a main component criterion for the diagnosis of FMS, the diagnosis of the FMS is still based on mostly patient's history and physical examination and there are no any confirmatory diagnostic tools such as blood tests (biomarkers), imaging, or histological analysis for FMS. Several investigations have clarified that muscle oxygenation changes, psychological, biochemical, hormonal and immunological factors are effective in the emergence of FMS, indicating that FMS should be considered as a complex disease that contains both environmental and genetic factors. ${ }^{1-3}$

Recent studies have strongly supported that the genetics may play an important role in the development of FMS and also in some other chronic musculoskeletal disorders. ${ }^{4}$ There is an eight-fold increased risk at the first-degree relatives of the patients with FMS and genetic studies have generated a number of candidate genes for FMS. ${ }^{1}$ However, these genetic studies have usually been in limited numbers and the results of the investigations are somewhat questionable. ${ }^{1}$ On the other hand, micro-ribonucleic acids (microRNAs) are short non-coding RNA molecules, approximately 19 to 25 nucleotides in size that may be involved in the regulation of the gene expression in the disease processes and physiological pathways. ${ }^{5}$ MicroRNAs regulate at least $30 \%$ of human genes and downregulate the gene expression from the target genes and recognize their target messenger RNAs based on the sequence complementarity. They inhibit the gene expression post-transcriptionally either by inhibition of translation or degradation of the target messenger RNA. ${ }^{5}$ MicroRNAs are also known to be key molecules, as they are effective in many vital biological processes, such as the cell proliferation and apoptosis. Since microRNAs can be detected in circulatory systems and other body fluids or in various tissues, they can be considered as potential biomarkers which may be used in the early diagnosis of many diseases. ${ }^{6}$ Recent studies have shown that microRNAs may have an important effect on the regulation of the pain processes in experimental models and clinical pain disorders. ${ }^{7-9} \mathrm{Li}$ et al. ${ }^{10}$ suggested that microRNAs might be associated with the pain pathophysiology of the osteoarthritis. Potential diagnostic and therapeutic properties of microRNAs have been demonstrated in patients with complex regional pain syndrome. ${ }^{11}$ The expression levels of microRNAs have also been searched in patients with FMS, and studies have revealed the presence of various microRNA expressions in patients' serum and cerebrospinal fluids (CSFs). Moreover, investigators have claimed that microRNAs might be useful in the development of biomarkers assessing disease activity, since some of the circulating microRNAs in patients with FMS was found to be in association with the pain severity. ${ }^{3,12}$ Mostly, studies have been focused on the relationship of circulating miRNAs only with the pain severity and physical functioning by using disease-specific questionnaires measuring generally functional health status of the patients; however, no data are available in the literature using more generic scales to reveal the association of plasma microRNA levels and the health-related quality of life (HRQoL) or general health status in patients with FMS. Therefore, further researches are essential not only for the identification of the potential confirmatory diagnostic biomarkers for the early definition of FMS or the assessment of disease activity in FMS, but also to find out for a possible correlation of the clinical characteristics related to the general health and HRQoL with the plasma level of microRNA expressions in patients with FMS.

The primary objective of this present study was to identify the plasma level of microRNA expressions in patients with FMS that might be a specific indicator for FMS as a confirmative diagnosis instrument of the disease. The secondary objective was to determine the relationship between the expression level of microRNAs in plasma with the general health and HRQoL together with the functional status in patients with FMS.

\section{PATIENTS AND METHODS}

This cross-sectional, case-control study was conducted at Mersin University Faculty of Medicine, Department of Physical Medicine and Rehabilitation between 2015 and April 2016. Thirty-five consecutive female patients with FMS (mean age: $42.0 \pm 11.8$ years; range, 21 to 62 years) 
who directly applied or were referred to our outpatient clinic and fulfilled the 1990 American College of Rheumatology (ACR) for FMS were included. The control group consisted of sex- and age-matched 35 healthy controls (mean age: $43.7 \pm 8.8$ years; range, 21 to 56 years) recruited from the general public and hospital staff. Patients were excluded from the study, if they had any inflammatory, metabolic, and neuromuscular disorder, severe infectious disease, malignancies, hepatic and renal disease or severe psychiatric disorder, serious comorbidities and cognitive impairment. None of the participants were immigrants, and individuals who were under the age of 18 and more than 65 years-old, pregnant, breastfeeding, and illiterate or did not have a sufficient mastery of Turkish language were also excluded. Due to the extremely limited numbers, data obtained from male patients were dismissed, as it would not be statistically conceivable to include them in the analysis, particularly while making sex comparisons. A written informed consent was obtained from each participant. The study protocol was approved by the Mersin University Faculty of Medicine Clinical Research Ethics Committee (No: 2015/216). The study was conducted in accordance with the principles of the Declaration of Helsinki.

\section{Clinical measurements}

Following the interview, all participants completed a form that described some of the demographical characteristics and clinical features of the FMS, including occupational, educational, and marital status, presence of fatigue, sleep disturbances, dysmenorrhea, depressive symptoms, morning stiffness, headache, upper and lower limb paresthesias, and symptoms of IBS. Careful medical history-taking and physical examination were carried out by a single clinician. During physical examination, positive sensitive point number (PSPN) was determined by palpation and body mass index (BMI) was noted. The patients were asked to fill the Fibromyalgia Impact Questionnaire (FIQ) pertaining to the functional status and the Medical Outcomes Study (MOS) 36-Item Short Form health survey (SF-36) scale for the evaluation of HRQoL under the supervision of investigators at the hospital.

The general health and functional status of the participants was assessed using the FIQ. The FIQ is one of the most widely used instruments for the evaluation of health status and outcome measures in patients with FMS. The scale is consisted of 20 items, in which 10 domains of FMS related symptoms and functional impairment: physical functioning, overall well-being, work day, pain, fatigue, morning tiredness, stiffness, job difficulty, depression and anxiety are being measured. The FIQ score ranges from 0 to 100 with high scores indicating severe functional impairment. ${ }^{13}$ The validity and reliability studies of the FIQ were conducted in the Turkish population. ${ }^{14}$ Only the total score of the FIQ scale was used for the statistical analyses.

The HRQoL of the participants were evaluated using the SF-36 scale. The SF-36 is a well-known generic questionnaire extensively used in clinical trials for the assessment of HRQoL. It includes a total of 36 questions which evaluate eight dimensions: physical functioning, role physical, pain index, general health, vitality, social functioning, role emotional, and mental health index with a summary measures of both physical and mental components. The score range of the questionnaire is from 0 up to 100 . High scores indicate better health status. ${ }^{15,16}$ The validity and reliability studies of the SF-36 were conducted in the Turkish population. ${ }^{17}$

Plasma collection and storage, RNA extraction, reverse transcription quantitative polymerase chain reaction ( $q P C R$ )

Serum samples of confirmative patients for FMS and healthy controls were obtained by the venipuncture of the cubital vein for the analysis of qPCR testing. Peripheral blood samples were initially collected into ethylenediaminetetraacetic acid (EDTA)-coated vacutainer tubes (BD, Vacutainer, Australia). Blood samples were processed after $2 \mathrm{~h}$ of the collection and the plasma separated by the centrifugation at $2,000 \times \mathrm{g}$ for $15 \mathrm{~min}$. The supernatants were, then, transferred into sterile centrifuge tubes either to be frozen at $-80^{\circ} \mathrm{C}$, or to be stored at $4^{\circ} \mathrm{C}$, until the further analysis for the instant microRNA extraction. All of the microRNAs observed in the study were extracted from fresh or frozen plasma samples by using the High Pure microRNA Isolation Kit (Roche Diagnostics $\mathrm{GmbH}$, Mannheim, Germany) according to the manufacturer's instructions. 
The reverse transcription reaction was, then, carried out via by the isolated RNA sample using the Qiagen miScript II RT Kit (Qiagen $\mathrm{GmbH}$, Hilden, Germany, Cat. No: 218161). The pre-amplification step reaction was performed to increase the quantity and the quality of cDNAs using the Qiagen miScript Microfluidics PreAMP Kit (Qigen $\mathrm{GmbH}$, Hilden, Germany, Cat No: $331455)$ before the qRT-PCR reaction. After the pre-amplification reaction had completed, the Exonuclease I reaction was applied to remove the non-specific signalizations of the deoxyribonucleic acid (DNA) molecule. Then, the microRNA analysis was enforced via by the high throughput real-time qPCR method using the BioMark HD System (Fluidigm Corporation, CA, USA). A total of 11 different microRNAs including miR-320a, miR-320b, miR-103a-3p, miR-107, let-7a-5p, miR-30b-5p, miR142-3p, miR-151a-5p, miR-374b-5p, miR-532-3p, and miR-939-5p were analyzed.

\section{Statistical analysis}

In the original study, the success performance for hsa-miR-320b in separating the patient and healthy group was 0.782 (area under the curve). Accordingly, 28 individuals in the patient group and 28 individuals in the control group were planned to be recruited (minimum $\mathrm{n}=56$ ) with $99 \%$ power and 5\% type I error. ${ }^{18}$ Considering the probability of a $20 \%$ loss rate in the data, 35 participants in each group and 70 participants in total were included in the study.

The results of microRNAs RT-PCR $(\mathrm{Ct}$ data) were normalized according to the global mean normalization strategy, as there are no verified housekeeping genes or universal controls in serum/plasma for the analysis of circulating microRNAs. Relative differences in microRNA expressions were determined by using the comparative threshold cycle $(\Delta \Delta \mathrm{CT})$ method, and fold change (FC) was calculated by the equation $2-\Delta \Delta$ Ct. $^{19,20}$ Statistical analysis was performed using the SPSS for Windows version 11.5 software (SPSS Inc., Chicago, IL, USA). The Shapiro-Wilk test was used to test the normal distribution of continuous measurements. Descriptive data were expressed in mean \pm standard deviation, median $\left(25^{\text {th }}-75^{\text {th }}\right.$ percentiles $)$ or number and frequency.
The Student's t-test and Mann-Whitney U test were used to compare the differences between two groups' means and medians. Correlations between continuous variables were evaluated with the Spearman's correlation coefficient (rho). Pearson's Chi-square test $\left(\chi^{2}\right)$ was used to assess the relationship between categorical variables in both groups. A $p$ value of $<0.05$ was considered statistically significant.

\section{RESULTS}

\section{Sociodemographic and baseline clinical} characteristics

Demographic and clinical characteristics of the patients and controls are shown in Table 1. There were no statistically significant differences in demographic and clinical characteristics between two groups in terms of age, BMI, marital status, and occupational and educational status. However, the PSPN in patients with FMS was found to be significantly higher than in controls $(p<0.001)$. Fatigue, sleep problems, dysmenorrhea, depressive symptoms, morning stiffness, headache, upper and lower limb paresthesia, and IBS symptoms were also statistically significantly more frequent in patients with FMS.

The subscales scores of the SF-36 questionnaire and the total FIQ scores of the patients and controls are summarized in Table 2 and Figure 1. Significant differences in correlations of all subscale scores of SF-36 between the groups, including the subscale of SF-36 physical symptom and mental symptom sum scores were found, and subscale scores of SF-36 significantly were lower in patients with FMS than in healthy controls (Table 1). In addition, a significant difference of the total FIQ score in patients with FMS was noted compared to the controls, indicating statistical significance $(\mathrm{p}<0.001$, Table 1).

Differences in microRNA expression levels between patients with FMS and healthy controls

A total of 11 plasma microRNAs-miR-320a, miR-103a-3p, miR-107, let-7a-5p, miR-30b-5p, miR-151a-5p, miR142-3p, miR-374b-5p, miR-320b, miR-532-3p, and miR-939-5p were found to be expressed in both groups. 


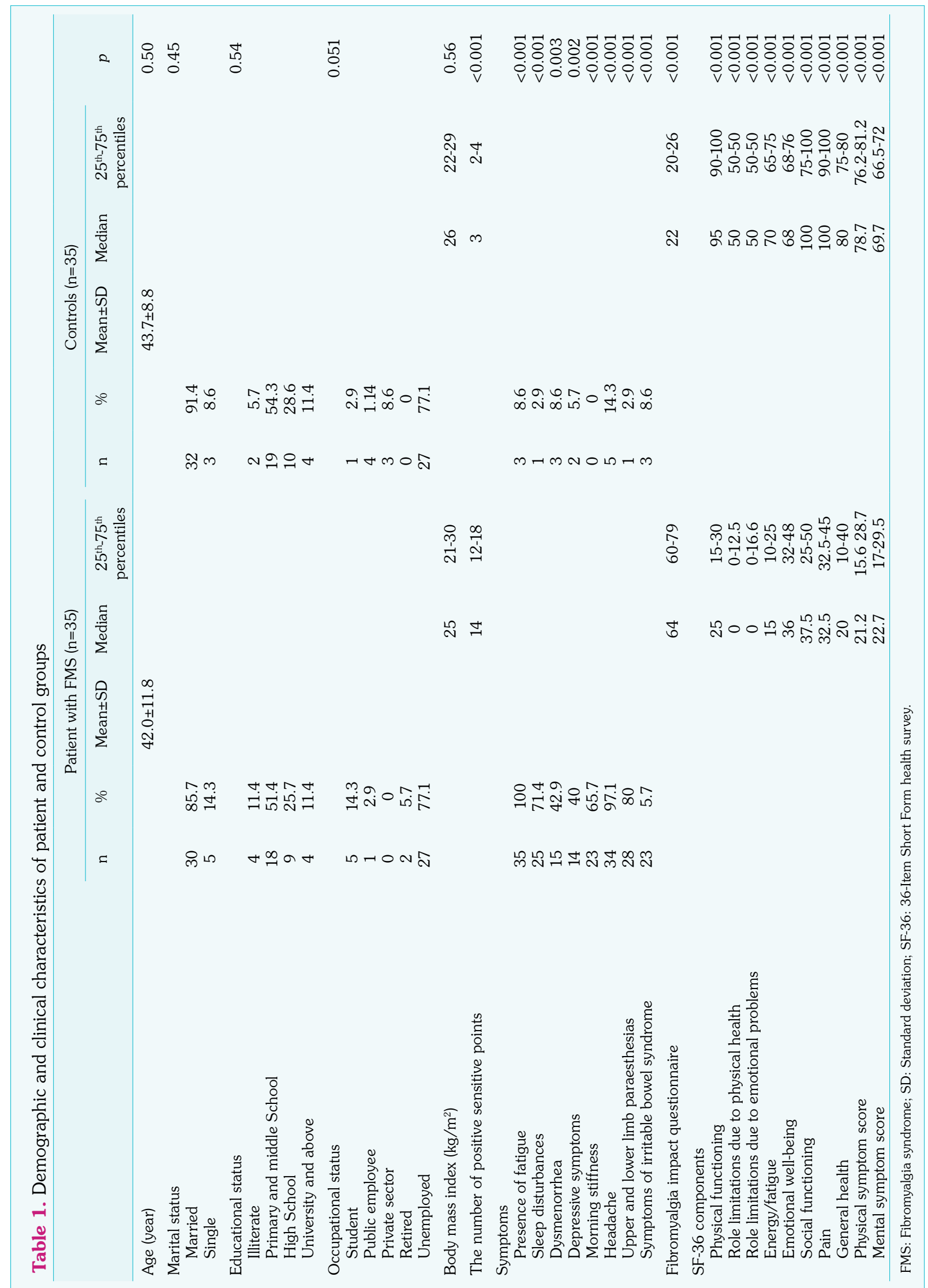


Table 2. Correlation of disease-associated microRNAs with common symptoms in FMS

\begin{tabular}{|c|c|c|c|c|c|c|c|}
\hline & & $\begin{array}{c}\mathrm{miR}-320 \mathrm{~b} \\
(\mathrm{n}=35)\end{array}$ & $\begin{array}{c}\mathrm{miR}-532-3 p \\
(\mathrm{n}=35)\end{array}$ & $\begin{array}{c}\mathrm{miR}-939-5 p \\
(\mathrm{n}=18)\end{array}$ & 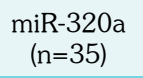 & $\begin{array}{c}\operatorname{miR}-103 a-3 p \\
(n=15)\end{array}$ & $\begin{array}{c}\mathrm{miR}-142-3 p \\
(\mathrm{n}=3)\end{array}$ \\
\hline \multirow{2}{*}{ FIQ } & $\mathrm{r}$ & -0.26 & -0.17 & 0.05 & -0.34 & 0.18 & 0.50 \\
\hline & $p$ & 0.14 & 0.32 & 0.83 & 0.05 & 0.51 & 0.67 \\
\hline \multirow{2}{*}{ BMI } & r & -0.20 & -0.07 & -0.18 & -0.29 & 0.17 & -0.50 \\
\hline & $p$ & 0.26 & 0.70 & 0.47 & 0.09 & 0.54 & 0.68 \\
\hline \multirow{2}{*}{ Height } & $\mathrm{r}$ & 0.15 & 0.23 & 0.27 & 0.36 & 0.29 & 0.50 \\
\hline & $p$ & 0.39 & 0.19 & 0.28 & 0.03 & 0.29 & 0.67 \\
\hline \multirow{2}{*}{ Weight } & $\mathrm{r}$ & -0.19 & -0.01 & -0.30 & -0.24 & 0.19 & -0.50 \\
\hline & $p$ & 0.27 & 0.96 & 0.22 & 0.17 & 0.50 & 0.67 \\
\hline \multirow{2}{*}{ PSPN } & r & 0.15 & 0.09 & -0.11 & 0.29 & -0.01 & 0.00 \\
\hline & $p$ & 0.38 & 0.60 & 0.68 & 0.09 & 0.97 & 1.00 \\
\hline \multirow{2}{*}{ Age } & $\mathrm{r}$ & -0.18 & -0.36 & -0.20 & -0.22 & 0.10 & -0.50 \\
\hline & $p$ & 0.30 & 0.03 & 0.43 & 0.20 & 0.73 & 0.68 \\
\hline \multicolumn{8}{|l|}{ SF-36 components } \\
\hline \multirow{2}{*}{ Physical functioning } & r & -0.43 & -0.17 & 0.03 & -0.43 & 0.03 & 0.50 \\
\hline & $p$ & 0.01 & 0.33 & 0.90 & 0.01 & 0.91 & 0.67 \\
\hline \multirow{2}{*}{$\begin{array}{l}\text { Role limitations due to } \\
\text { physical health }\end{array}$} & r & 0.07 & 0.11 & 0.07 & 0.08 & -0.07 & -0.90 \\
\hline & $p$ & 0.69 & 0.54 & 0.80 & 0.64 & 0.80 & 0.33 \\
\hline \multirow{2}{*}{$\begin{array}{l}\text { Role limitations due to } \\
\text { emotional problems }\end{array}$} & r & -0.05 & -0.10 & -0.04 & -0.10 & -0.12 & NA \\
\hline & $p$ & 0.77 & 0.56 & 0.88 & 0.58 & 0.66 & NA \\
\hline \multirow{2}{*}{ Energy/fatigue } & r & -0.07 & -0.09 & -0.18 & -0.12 & -0.16 & 0.00 \\
\hline & $p$ & 0.68 & 0.62 & 0.94 & 0.48 & 0.58 & 1.00 \\
\hline \multirow{2}{*}{ Emotional well-being } & r & -0.24 & -0.22 & 0.18 & -0.28 & -0.16 & 0.50 \\
\hline & $p$ & 0.17 & 0.20 & 0.47 & 0.10 & 0.56 & 0.67 \\
\hline \multirow{2}{*}{ Social functioning } & $\mathrm{r}$ & -0.12 & -0.06 & 0.12 & -0.27 & -0.04 & 0.50 \\
\hline & $p$ & 0.49 & 0.74 & 0.64 & 0.11 & 0.90 & 0.67 \\
\hline \multirow{2}{*}{ Pain } & $\mathrm{r}$ & -0.17 & 0.03 & 0.03 & -0.28 & -0.33 & 0.87 \\
\hline & $p$ & 0.33 & 0.85 & 0.89 & 0.11 & 0.23 & 0.67 \\
\hline \multirow{2}{*}{ General health } & r & 0.12 & 0.29 & 0.46 & 0.03 & 0.21 & 0.00 \\
\hline & $p$ & 0.48 & 0.89 & 0.05 & 0.85 & 0.45 & 1.00 \\
\hline \multirow{2}{*}{ Physical symptom score } & r & -0.10 & 0.11 & 0.31 & $\mathrm{r}-0.17$ & 0.05 & 0.50 \\
\hline & $p$ & 0.56 & 0.54 & 0.22 & 0.34 & 0.86 & 0.67 \\
\hline \multirow{2}{*}{ Mental symptom score } & r & -0.15 & -0.16 & 0.02 & -0.23 & -0.17 & 1.00 \\
\hline & $p$ & 0.41 & 0.35 & 0.94 & 0.19 & 0.55 & $<0.001$ \\
\hline
\end{tabular}

FMS: Fibromyalgia syndrome; FIQ: Fibromyalgia Impact Questionnaire; BMI: Body mass index; PSPN: Positive sensitive point number; SF-36: 36-Item Short Form health survey.

No any significant differences were detected among these 11 plasma levels of expressed microRNAs (miR-320a, miR-103a-3p, miR-107, let-7a-5p, miR-30b-5p, miR-151a-5p, miR142-3p, miR-374b-5p, miR-320b, miR-532-3p, and miR-939-5p) between the patients with FMS and healthy controls. No statistical FC was recorded in the expression of 11 plasma microRNA levels in patients with FMS (Figure 2).
MicroRNAs and quality of life, and general health and functional status

Correlations between the 11 expression levels of microRNAs in plasma, demographic clinical data, the general health and functional status, and the HRQoL in patients with FMS were assessed by using the FIQ and the SF-36 scores, respectively. However, out of 11 expressed microRNAs in patients' plasma, a total of 


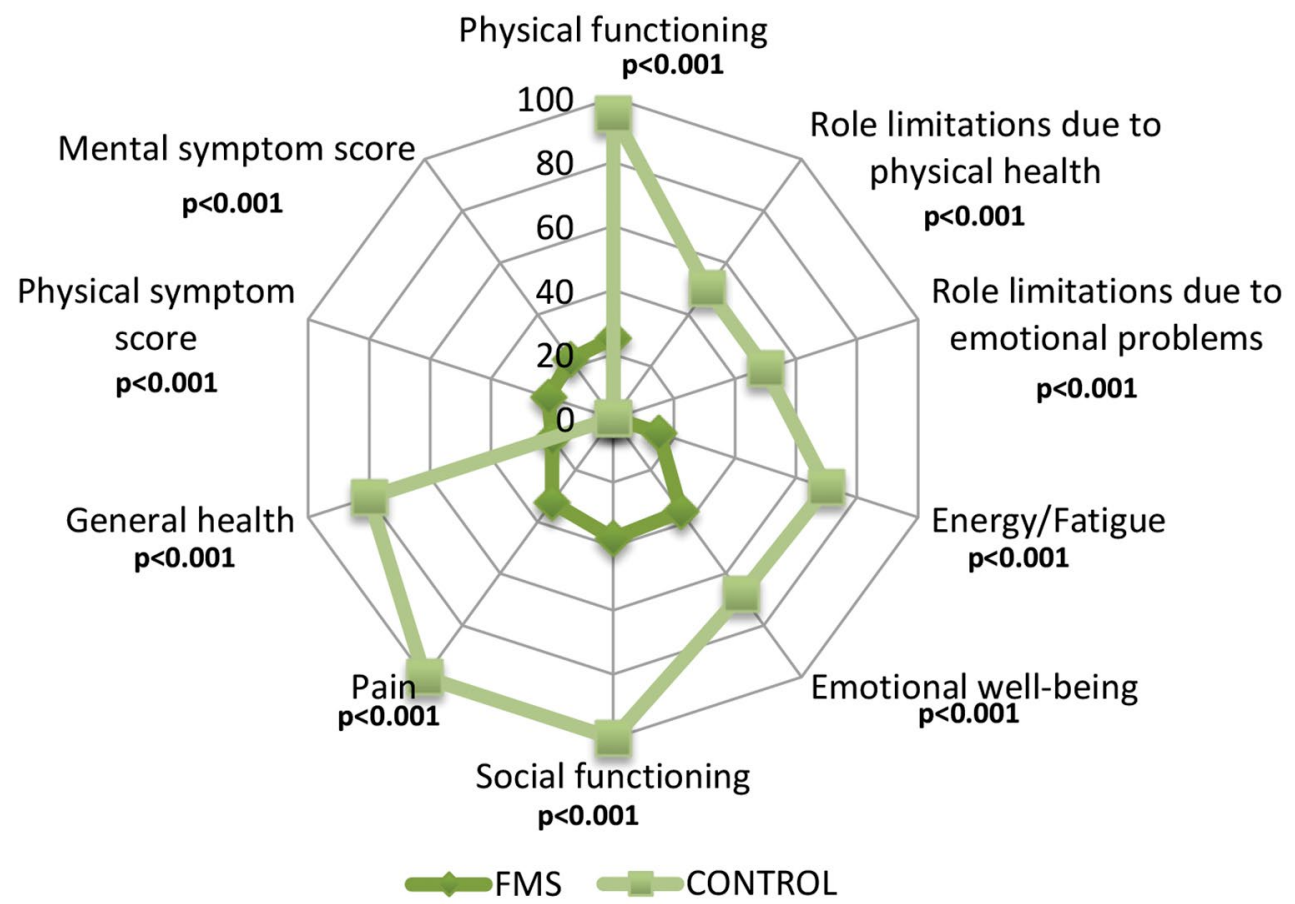

Figure 1. Comparison of SF-36 components in the FMS and control groups. SF-36: 36-Item Short Form health survey; FMS: Fibromyalgia syndrome.

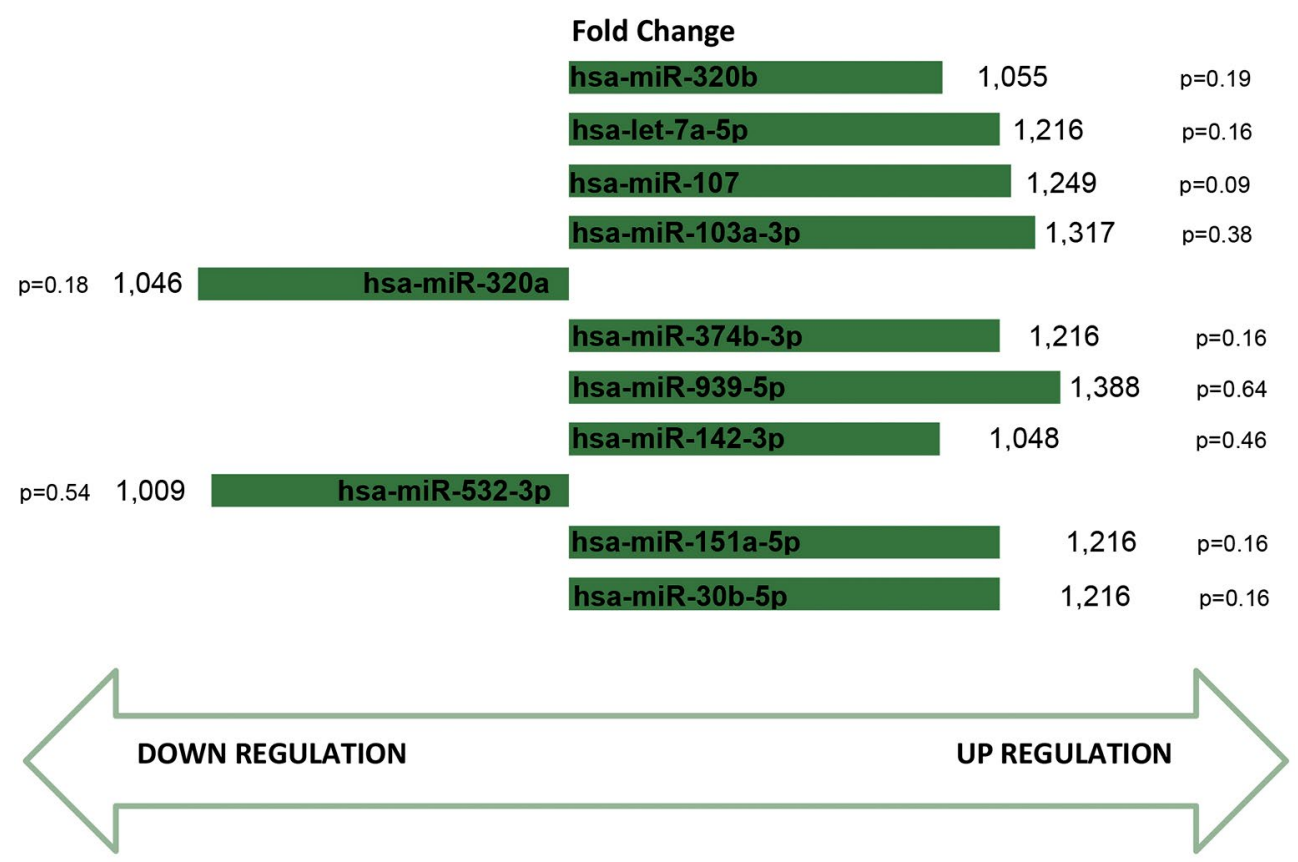

Figure 2. Comparison of microRNA expressions in the FMS and control groups. microRNA: Micro-ribonucleic acids; FMS: Fibromyalgia syndrome. 
five microRNAs-miR-151a-5p, miR-374b-3p, miR-107, let-7a-5p, and miR-30b-5p - were removed from the statistical analysis, since their plasma expressions were not sufficiently in comparable levels to attain a correlation value, and only the plasma expression levels of the six remaining microRNAs (miR-320b, miR-532-3p, miR-939-5p, miR-320a, miR-103a-3p, miR-142-3p) were included in statistical analysis. Weak negative correlations were observed between the expression level of miR-532-3p in plasma and the mean age $(\mathrm{r}=-0.36, \mathrm{p}=0.03)$ of the patients, and between the plasma miR-320a expression level and the total score of the FIQ scale $(\mathrm{r}=-0.34, \mathrm{p}=0.05)$ in patients with FMS, whereas the mean height of the patients with FMS was positively correlated with the plasma miR-320a expression level $(\mathrm{r}=0.36, \mathrm{p}=0.03)$. No relationship between the expression levels of the six remaining microRNAs (miR-320b, miR-532-3p, miR-939-5p, miR-320a, miR-103a-3p, and miR-142-3p) in plasma and the means of weight, BMI, and PSPN was found in patients with FMS. A negative correlation between the subscale score of SF-36 physical functioning and the expression levels of miR-320a and $\mathrm{miR}-320 \mathrm{~b}$ in plasma were $(\mathrm{p}=0.01, \mathrm{r}=-0.43$ and $p=0.01, r=-0.43$, respectively). Patients with lower miR-320a and miR-320b expression levels had higher SF-36 physical function scores. The SF-36 physical function scores increased, as the expression level decreased. A strong positive correlation was found between miR-142-3p and the subscale score of SF-36 mental symptom score in female patients with FMS $(p<0.001$, $r=1.00$ ). No any other significant relationship was noted ( $p>0.05$ ) between the other subscales scores of SF-36, including pain, role physical, role emotional, general health, vitality, social functioning, mental health, and sum scores of physical functions, and the plasma expression levels of microRNAs (miR-532-3p, miR-939-5p, and miR-103a-3p) in patients with FMS (Table 2).

\section{DISCUSSION}

Fibromyalgia syndrome is a chronic musculoskeletal disease with an unknown etiology, characterized by widespread body pain and decreased pain threshold and the underlying pathophysiological mechanism of the disease is still to be elucidated. ${ }^{1,2}$

The verification of the extracellular microRNAs family existing in various biological fluids of the body (the circulating microRNAs) in numerous studies has led to a global consideration among the investigators indicating that microRNAs might be accepted as potential biological markers for the diagnosis or disease activity of several disorders, including also the FMS. MicroRNAs was initially identified by Bjersing et al. ${ }^{12}$ in the CSF of 10 female patients with FMS and the expression of nine microRNAs (miR-21-5p, miR-145-5p, miR-29a-3p, miR-99b-5p, miR-125b-5p, miR-23a-3p, 23b-3p, miR-195-5p, miR-223 $3 p)$ were found to be significantly in low levels, compared to healthy controls. A total of 374 serum expression levels of microRNAs in 20 female patients with FMS and 20 healthy female controls were also studied subsequently by the same investigators. ${ }^{3}$ The results revealed that serum levels of eight microRNAs in patients with FMS were measured in differently expressed, in which seven out of the eight microRNAs (miR-103a-3p, miR-107, miR-let-7a-5p, miR-30b-5p, miR-151a-5p, miR-142-3p and miR-374b-5p) were significantly in low levels, while only the serum expression level of miR-320 was detected significantly higher than healthy individuals. Similarly, Leinders et al. ${ }^{21}$ screened the expression of 51 dysregulated white blood cell microRNAs, including miR-let-7d, miR-103 and miR-151 in patients with FMS and demonstrated a functional impairment of the small nerve fibers (A-delta and C fibers) and a significant increase of miR-let-7d levels in skin biopsies of the patients with FMS. Furthermore, downregulation of a large subset of microRNA (about 20\% of the 1212 sequences analyzed), particularly lower expression levels of miR-223-3p, miR-451a, miR-338-3p, miR-143-3p, miR-145-5p and miR-21-5p in peripheral blood mononuclear cells (PBMCs) of 11 female patients with the FMS was reported by Cerdá-Olmedo et al. ${ }^{19}$ Interestingly, the low expression levels of miR-223-3p, miR-145-5p and miR-21-5p in PBMCs that have been reported previously in CSF of the patients with FMS by Bjersing et al. $^{3}$ have also been demonstrated in this 
study, although the reason for this could not be explained. Moreover, a recent study conducted by Masotti et al. ${ }^{18}$ analyzed the panel of 179 circulating microRNAs profile in serum and saliva of 14 female patients newly diagnosed as FMS compared to those sex-and age-matched healthy controls, where the significant decrease of six microRNA serum expression levels (miR-23a-3p, miR-1, miR-133a, miR-346, miR-139-5p and miR-320b) were noted. Serum expression level of miR-23, which was demonstrated in previous studies with a low level in CSF of the patients with FMS, was found to decrease, as well in their study. Nonetheless, the data derived from the low expression levels of these six microRNAs in serum were subsequently compared to the saliva samples of the patients with FMS, and no significant differences of microRNAs expressions in saliva levels between the patient and control groups have been reported. ${ }^{18}$ In general, results from all these studies have induced a bias that low expression levels of microRNAs, detected in various body fluids of the patients with FMS could be defined as a distinctive entity for the differential diagnosis of the FMS in clinical practices. However, in contrast to these previously reported studies, we observed no significant changes in patients with FMS in terms of the expression levels of 11 extracted microRNAs in plasma, compared to healthy controls. One of the plausible explanations for this conflicting result of our study is the major insufficiency of the patient selection criteria, as patients with FMS using analgesics or selective serotonin or norepinephrine reuptake inhibitor drugs (i.e., paracetamol or duloxetine hydrochloride) were also enrolled in the patient group without no cessation of the drug use prior to the study. Although we cannot clarify entirely to what extent drugs have influences on the pathophysiological mechanism due to the ambiguous onset of the FMS, the endogenous expression of circulating microRNA levels may be seriously affected by the pharmacological treatment in patients with FMS. ${ }^{20}$ Additionally, the unknown status of patients with severe viral infections in cohorts, as they were mostly neglected during the patient selection procedure of previous investigations, may be also a major consideration for the effectiveness on the low expression levels of the circulating microRNAs in patients with FMS, and as a consequence may modify the results of the investigations. ${ }^{22}$
Although no confirmative data regarding the isolation of a viral microorganism associated specifically to FMS has been published to date, the etiological role of viral infections should not be entirely rejected in FMS. ${ }^{1}$ Various results of a limited number of studies have demonstrated that no correlations or overlaps between the different body tissues or fluids related to the expression levels of microRNAs were detected and, therefore, it is highly reasonable to speculate that the expression levels of microRNAs may be unstable depending on the body tissues and biological fluids in patients with FMS. Moreover, the heterogeneity of the technical methods used in the measurement for the identification of accurate dysregulation in circulating microRNAs expression levels, the presence of microRNAs profile itself with an enormous spectrum in various biological components of the human body, and the uncertainty of the current disease activity status for FMS in different biological fluids, cells or tissues may also alter the expression levels of microRNAs within these biological components in patients with FMS. ${ }^{23}$ Obviously, biopsy samples obtaining from the various human body components should be taken into consideration for future investigations as a remarkable parameter to classify the expression levels of microRNAs in different biological compounds.

The secondary objective of this present study was to search for the correlation of the expression levels of microRNAs in plasma with the general health status and physical functioning and HRQoL in patients with FMS by using SF-36 and FIQ scales, since the latter is one of the most frequently used tools for the evaluation of general health and functional status specifically in patients with FMS. Investigations revealing the relationship between the expression levels of microRNAs with the HRQoL and the general health and functional status or symptom severity in patients with FMS are rather lacking and findings are highly variable and controversial. A positive significant correlation between the miR-145-5p expression level in CSF and the FIQ pain and fatigue scores in patients with FMS has already been demonstrated. ${ }^{12}$ However, the FIQ pain score was negatively correlated with the miR-320a serum expression in a clinical trial, while the expression of serum miR-103a-3p, miR-30b-5p and miR-374b-5p levels were positively correlated with the FIQ pain 
and sleep amount scores. ${ }^{3}$ On the other hand, no significant correlation between the total FIQ score and the expression levels of microRNAs in plasma was reported by Cerdá-Olmedo et al. ${ }^{19}$ In addition, the authors noted a possible relationship between some of the expressed levels of microRNAs in PBMCs and the severity of the mental fatigue, using multidimensional fatigue inventory scale. However, our findings were incompatible with the findings of Cerdá-Olmedo et al., ${ }^{19}$ but consistent with the FIQ pain score, detected by Bjersing et $a .,{ }^{3}$ as they showed a negative correlation ( $p=0.049, r=-0.336$ ) between the expression level of $\mathrm{miR} 320 \mathrm{a}$ in plasma and the total FIQ score indicating that the high score of total FIQ scale may result in downregulation of the miR320a in patients with FMS.

The relationship between six microRNAs level expressed in plasma and the HRQoL was assessed using the SF-36 scale in patients with FMS in our study. Among these expressed microRNAS in plasma, the level of miR-320a and together with miR-320b was found to be negatively correlated with the subscale of SF-36 physical functioning score which is similar to our finding of correlation with the total FIQ score. On the contrary, a strong positive correlation was found between miR-142-3p and the subscale score of SF-36 mental symptom score in female patients with FMS compatible with previous investigations, where the cardinal symptoms of FMS, including pain and fatigue assessed by subscale of FIQ measurement and, the depression evaluated by Zung Self-Rating Depression Scale were found to be in correlation with the dysregulated expression levels of microRNAs. ${ }^{18}$ Furthermore, to the best of our knowledge, this is the first study to analyze the correlation of the SF-36 scale and the expression levels of microRNAs in plasma in patients with FMS, and such an association has never been reported before in the literature. Surprisingly, no any other significant relationship between the subscale scores of SF-36 pain, SF vitality, and sum scores of SF-36 physical functions, and the expression levels of microRNA in plasma were observed in our study. This may be due to the fact that in contrary to FIQ scale, the SF-36 is a generic questionnaire that has the advantage of being more psychometrically sound and the ability of measuring the functional or health status with multi-dimension. On the other hand, the FIQ measurement is more likely a disease specific questionnaire that takes the advantage on domains related to what is being measured. Additionally, the lack of data from the item or domain scores in FIQ scale, which may reflect the relationship between some of the cardinal symptoms of FMS with the expression levels of microRNAs in plasma, should be considered a limitation of the present study, as only the total FIQ score of the patients was used in statistical analyses. However, special caution should be exercised while interpreting these results due to the relatively low sample size of our cohorts for wide comparative analysis in epigenetic studies (not recommended less than 100 individuals in every group. ${ }^{24}$ The sex selection and the inability of the evaluation for causative agents due to the cross-sectional design of the study impede the generalizability of our findings.

In conclusion, although it is difficult to draw definite conclusions, our findings suggest that it is too early to consider the expression levels of microRNAs in plasma as a confirmative biological instrument in patients with FMS. Moreover, both disease-specific questionnaires and generic scales, the SF-36 in particular should be more involved in clinical trials, while comparing the expression levels of the circulating microRNAs with the key symptoms and the disease severity or the general health and functional status, and the HRQoL in patients with FMS. Considering the wide alteration in circulating microRNA expression levels, the tissue- specific prospective studies relevant to biopsy samples obtaining from the various human body components with the adequate sample size in study groups are essential to classify the expression levels of microRNAs in patients with FMS.

\section{Declaration of conflicting interests}

The authors declared no conflicts of interest with respect to the authorship and/or publication of this article.

\section{Funding}

This study was supported by Mersin University Scientific Research Projects Unit with project number 2015-TP3-1181. 


\section{REFERENCES}

1. Häuser W, Ablin J, Fitzcharles MA, Littlejohn G, Luciano JV, Usui C, et al. Fibromyalgia. Nat Rev Dis Primers 2015;1:15022.

2. Araújo FM, DeSantana JM. Physical therapy modalities for treating fibromyalgia. F1000Res 2019;8:F1000 Faculty Rev-2030.

3. Bjersing JL, Bokarewa MI, Mannerkorpi K. Profile of circulating microRNAs in fibromyalgia and their relation to symptom severity: An exploratory study. Rheumatol Int 2015;35:635-42.

4. Diatchenko L, Fillingim RB, Smith SB, Maixner W. The phenotypic and genetic signatures of common musculoskeletal pain conditions. Nat Rev Rheumatol 2013;9:340-50.

5. Niederberger E, Kynast K, Lötsch J, Geisslinger G. MicroRNAs as new players in the pain game. Pain 2011;152:1455-8.

6. Kynast KL, Russe OQ, Geisslinger G, Niederberger E. Novel findings in pain processing pathways: implications for miRNAs as future therapeutic targets. Expert Rev Neurother 2013;13:515-25.

7. Shenouda SK, Alahari SK. MicroRNA function in cancer: Oncogene or a tumor suppressor? Cancer Metastasis Rev 2009;28:369-78.

8. Lutz BM, Bekker A, Tao YX. Noncoding RNAs: New players in chronic pain. Anesthesiology 2014;121:409-17.

9. Alevizos I, Illei GG. MicroRNAs as biomarkers in rheumatic diseases. Nat Rev Rheumatol 2010;6:391-8.

10. Li X, Kroin JS, Kc R, Gibson G, Chen D, Corbett GT, et al. Altered spinal microRNA-146a and the microRNA-183 cluster contribute to osteoarthritic pain in knee joints. J Bone Miner Res 2013;28:2512-22.

11. Orlova IA, Alexander GM, Qureshi RA, Sacan A, Graziano A, Barrett JE, et al. MicroRNA modulation in complex regional pain syndrome. $\mathrm{J}$ Transl Med 2011;9:195.

12. Bjersing JL, Lundborg C, Bokarewa MI, Mannerkorpi K. Profile of cerebrospinal microRNAs in fibromyalgia. PLoS One 2013;8:e78762.

13. Burckhardt CS, Clark SR, Bennett RM. The fibromyalgia impact questionnaire: development and validation. J Rheumatol 1991;18:728-33.

14. Sarmer S, Ergin S, Yavuzer G. The validity and reliability of the Turkish version of the Fibromyalgia Impact Questionnaire. Rheumatol Int 2000;20:9-12.
15. Ware JE Jr, Kosinski M, Bayliss MS, McHorney CA, Rogers WH, Raczek A. Comparison of methods for the scoring and statistical analysis of SF-36 health profile and summary measures: summary of results from the Medical Outcomes Study. Med Care 1995;33(4 Suppl):AS264-79.

16. Ware JE Jr, Sherbourne CD. The MOS 36-item shortform health survey (SF-36). I. Conceptual framework and item selection. Med Care 1992;30:473-83.

17. Kocyigit $\mathrm{H}$. Reliability and validity of the Turkish version of short form-36 (SF-36): A study in a group of patients will rheumatic diseases. Turk J Drugs Ther 1999;12:102-6.

18. Masotti A, Baldassarre A, Guzzo MP, Iannuccelli C, Barbato C, Di Franco M. Circulating microRNA profiles as liquid biopsies for the characterization and diagnosis of fibromyalgia syndrome. Mol Neurobiol 2017;54:7129-36.

19. Cerdá-Olmedo G, Mena-Durán AV, Monsalve V, Oltra E. Identification of a microRNA signature for the diagnosis of fibromyalgia. PLoS One 2015;10:e0121903.

20. Almenar-Pérez E, Sánchez-Fito T, Ovejero T, Nathanson L, Oltra E. Impact of polypharmacy on candidate biomarker mirnomes for the diagnosis of fibromyalgia and myalgic encephalomyelitis/chronic fatigue syndrome: Striking back on treatments. Pharmaceutics 2019;11:126.

21. Leinders M, Doppler K, Klein T, Deckart M, Rittner $\mathrm{H}$, Sommer $\mathrm{C}$, et al. Increased cutaneous miRlet-7d expression correlates with small nerve fiber pathology in patients with fibromyalgia syndrome. Pain 2016;157:2493-503.

22. Gaulke CA, Porter M, Han YH, Sankaran-Walters S, Grishina I, George MD, et al. Intestinal epithelial barrier disruption through altered mucosal microRNA expression in human immunodeficiency virus and simian immunodeficiency virus infections. J Virol 2014;88:6268-80.

23. Petty RD, McCarthy NE, Le Dieu R, Kerr JR. MicroRNAs hsa-miR-99b, hsa-miR-330, hsa-miR-126 and hsa-miR-30c: Potential diagnostic biomarkers in Natural Killer (NK) cells of patients with Chronic Fatigue Syndrome (CFS)/ Myalgic Encephalomyelitis (ME). PLoS One 2016;11:e0150904.

24. Polli A, Godderis L, Ghosh M, Ickmans K, Nijs J. Epigenetic and miRNA Expression Changes in People with Pain: A Systematic Review. J Pain 2020;21:763-80. 